\title{
Plasma-spray Synthesis and Characterization of Ti-based Nitride and Oxide Nanogranules
}

\author{
G. S. E. Antipas* \\ School of Mining Engineering and Metallurgy, National Technical University of \\ Athens, Zografou Campus, Athens 15780, Greece
}

Received: November 13, 2013; Revised: July 24, 2014

\begin{abstract}
The synthesis of nanosized Ti-based nanogranules via plasma spraying is reported. The synthesis route involved use of both nitrogen and oxygen gases with varying results. In the case of nitrogen, a mixture of titanium nitrides were produced, yielding both the $\mathrm{Ti}_{2} \mathrm{~N}$ and the sub-stoichiometric $\mathrm{TiN}_{0.61}$ compounds. In the case of oxygen, both the stoichiometric rutile and $\mathrm{TiO}$ ceramic phases were indexed. Based on EDS analysis, even fractional oxygen concentrations caused tungsten impurities which originated from the cathode electrode. The method yielded particle mass median sizes of the order of $15 \mathrm{~nm}$ and the smallest particles detected were $5 \mathrm{~nm}$.
\end{abstract}

Keywords: $\mathrm{TiN}, \mathrm{TiO}$, plasma spraying, rapid quenching

\section{Introduction}

Titanium nitrides are low-cost coating materials of choice owing to their enhanced tribology and biocompatibility ${ }^{1,2}$. Notable nanoscale titanium nitride applications are diverse, such as catalysts increasing durability in proton exchange membrane fuel cells ${ }^{3}$, prosthetic materials in total hip and knee replacements ${ }^{4}$ and utilization in medical tools ${ }^{5}$ such as vascular stents ${ }^{6}$ and, lately, plasmonic materials with improved optical properties in the visible and near-infrared regions ${ }^{7,8}$. On the other hand, titanium oxides have also found very practical applications such serving as active coatings in self-cleaning window products ${ }^{9}$ but are probably best known for their photocatalytic ${ }^{10}$ and photoluminescence ${ }^{11}$ properties; in particular, titanium oxide nanocrystals embedded in alumina have been lately applied in ultraviolet photocatalysis 9 .

The simultaneous synthesis of nanostructured titanium nitrides and oxides at relatively high production rates at controlled stoichiometries is a challenge. Nanostructured materials exhibit enhanced properties compared to the polycrystalline phase ${ }^{12}$. For example, although metallic nanostructured alloys may be sintered at low temperatures they, nonetheless, show higher hardness and yield strength than polycrystalline equivalents ${ }^{13}$. There are various techniques available for making nm-sized particles and they invariably address the degree of agglomeration when bulk nanostructures or nm dispersions are desired ${ }^{14}$. Also, in order to manufacture nanostructured materials, it is necessary to control the particle size and chemical stoichiometry at the same time and this can typically by achieved by rapid solidification ${ }^{15}$, albeit not in a large (e.g. industrial) scale.

One of the alternative routes for increased production rates of nanosized materials may be through plasma

*e-mail: gantipas@metal.ntua.gr spraying ${ }^{16}$, in which an arc generated torch achieves vaporization of a high purity precursor material. In this process, the interior of a chamber is maintained at a pressure of up to $20 \mathrm{kPa}$ while the precursor material is vaporized by a non-consumable tungsten electrode shielded by a blanket of inert gas, the latter both protecting the electrode from oxidation and maintaining the plasma arc. The inert gas phase is directed into the metallic vapour by a water cooled nozzle attached to the torch. The consumable precursor material is in the form of a rod, typically with a diameter up to $5 \mathrm{~cm}$, fed horizontally or vertically relative to the non-consumable electrode; it is also electrically grounded and cooled by a copper anode and able of both translational and rotational motion. Homogeneous gas injection is achieved by the use of closecoupled gas injection nozzles ${ }^{17-19}$. However, with increasing production rates (and, hence, with increasing vapour density ${ }^{19}$ ), both the gas flow rate and location of injection become important. The reaction gas can be introduced either together with the quench gas or separately in order to form an oxide, carbide or nitride from the metallic or semiconducting precursor material.

Introducing the reaction gas with the quench gas into the plasma allows the synthesis of a higher melting-point material, which enhances quenching and facilitates formation of nanocrystal particles. Also, by using a dissociable gas, for example, $\mathrm{H}_{2}$ or $\mathrm{N}_{2}$, along with the working gas allows the plasma to reach a higher temperature, producing a more complete reaction of the precursor vapour. Here, a number of Ti-based nanogranules are produced by plasma spraying with nitrogen and oxygen mixtures and their properties are discussed in terms of the main operating variables of the process with the aim of highlighting aspects of the effect of the flowrate and type of atomizing gas injected into the plasma reaction zone on granule size. 


\section{Material and Methods}

In separate experiments, titanium metal vapour produced by plasma spraying was brought in contact with nitrogen and oxygen gases toward formation of $\mathrm{TiN}$ and $\mathrm{TiO}_{2}$ nanogranules, respectively. The plasma spray facility used in this study is shown in Figure 1. The facility comprised four main control volumes. The upper volume (Figure 1a) contained the plasma spray and atomizer setup and consisted of the plasma arc and the gas injection nozzles, where introduction of the atomizing gas was at an angle from the vertical direction. The first stage of the process consisted of vaporization of a Ti rod by a tungsten inert gas (TIG) wrapped in a stream of Argon and cooled by a continuous flow of water, under ambient pressure conditions. The $\mathrm{Ti}$ rod was cooled by a $\mathrm{Cu}$ plate anode (see Figure 1a). The Argon flow shields against oxidation of the non consumable tungsten electrode and, further downstream, serves as an ionizing medium for the creation of the plasma arc. Introduction of the atomizing assembly was at an angle from the vertical direction so as to achieve an elongated plasma tail. Both Nitrogen and Oxygen flows were used as the quench/reaction medium. Both the quench/reaction and working gas flows were monitored by flow meters and were constantly regulated.
Upon atomization, gas expansion induced further cooling of the two-phase flow ${ }^{19}$; the expanding turbulent flow was then confined by a collecting cone (see Figure 1b) which was intended to hinder the material's in-flight agglomeration.

The two phase flow was then continuously recirculated between the collecting cone (Figure 1b) and the adjacent filtering compartment (see Figure 1c) by an ambient air flow maintained by a fan blower. The purpose of this stage was to withhold weakly segregated nanoparticles, separating them from the rest of the flow, which then consisted of nitride (oxide) powders. The latter were then routed into a storage compartment (see Figure 1d) through a collecting cone. A close up schematic of the plasma spraying nozzle is shown in Figure 1d.

The most critical operational parameters of the process were the point of gas injection and the degree of dilution of the nanocrystal aerosol. These parameters affected average particle size, size distribution and the degree of agglomeration.

A number of experiments were carried out for the determination of the effect of the gas flowrate on the size of Ti particles in a nitrogen flow. In addition, two nanocrystal synthesis experiments were performed for the study of particles via electron microscopy. In the latter experiments,

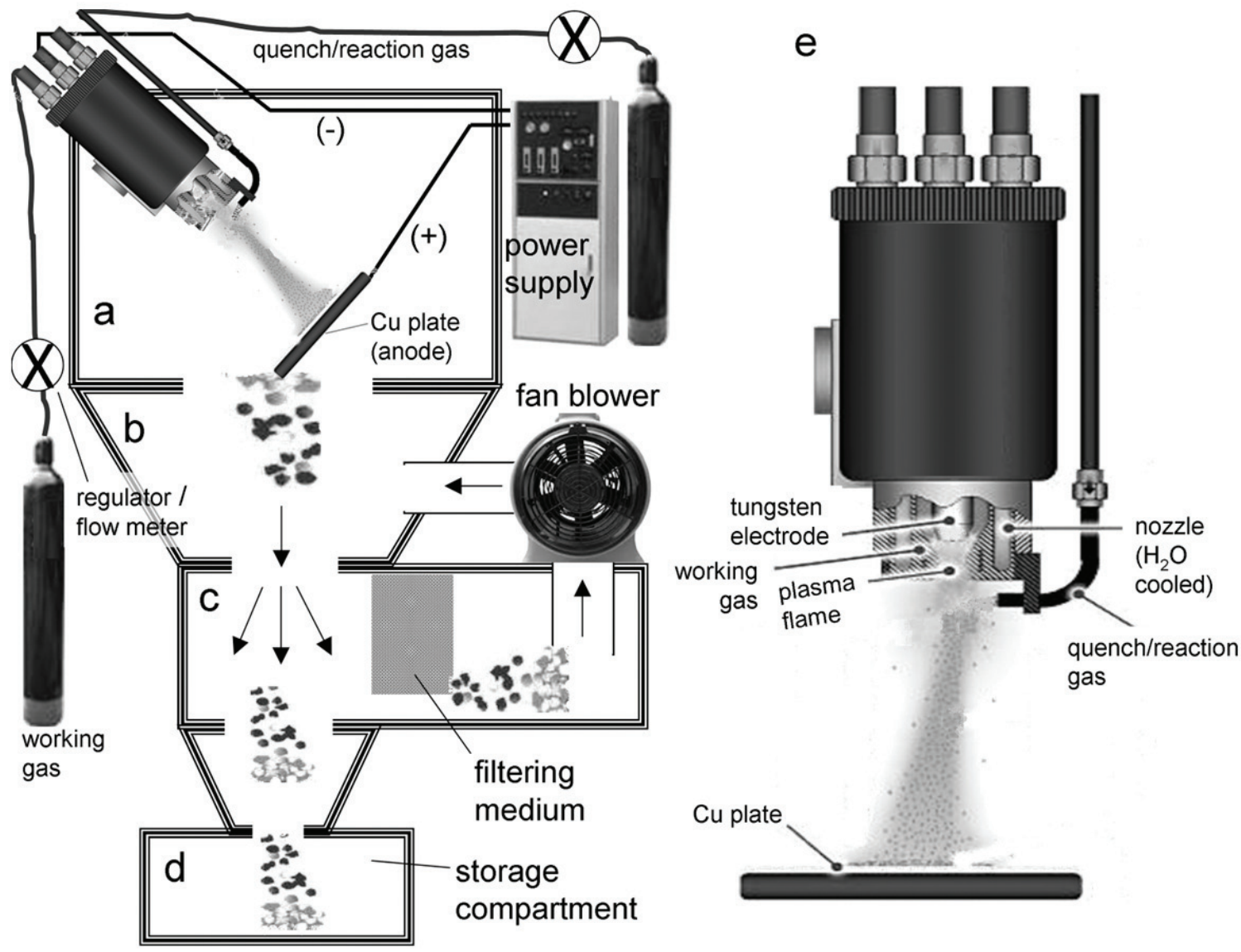

Figure 1. Schematic of the plasma spray setup. a) Plasma arc and atomizer, b) powder collecting cone, c) air recirculating and filtering compartment, d) storage compartment and d) close up view of the atomizer. 
a metallic Ti rid with a diameter of $7.5 \mathrm{~cm}$ (purity 99.99\%) was used as the arc anode. Nitrogen gas injected at a rate of $14 \mathrm{~m}^{3} / \mathrm{h}$ interacted with the Ti metal vapour towards formation of nitrides. Quench $\mathrm{N}_{2}$ gas at a flux of $36 \mathrm{~m}^{3} / \mathrm{h}$ was then injected to further cool the particles and prevent hard agglomeration. In a second experiment, Ti metal powder was mixed in a $50 \mathrm{wt} . \%$ ratio with Ti oxide powder. The powders were then compacted into a rod $7.5 \mathrm{~cm}$ in diameter by pressing and sintering. The rod was electrically conductive and used as an anode in a transferred arc. The anode was evaporated by the arc and the anode vapour was injected at an approximate rate of $200 \mathrm{~g} / \mathrm{h}$ into the plasma. Following this, oxygen was injected into the plasma at a flowrate of $35 \mathrm{~m}^{3} / \mathrm{h}$ to mediate cooling, finally yielding metal-oxide ceramic particles. Quench nitrogen gas $\left(30 \mathrm{~m}^{3} / \mathrm{h}\right)$ was later added to further cool the particles and prevent hard agglomeration. In both experiments, the cathode was a tungsten electrode shielded by a flow of $4 \mathrm{~m}^{3} / \mathrm{h}$ of argon working gas combined with nitrogen and hydrogen at a volume ratio of $2: 2: 1$ respectively. The applied electric current was $750 \mathrm{~A}$.
X-ray diffraction (XRD) spectra were recorded on a Bruker D8-Focus diffractometer with nickel-filtered $\mathrm{Cu}$ Ka radiation $(\lambda=1.5405 \AA)$, operating at $40 \mathrm{kV}$ and 40 mA. Sample cross sections were examined by scanning electron microscopy (SEM) on a Jeol 6380LV SEM, which also recorded energy dispersive X-ray spectra (EDS). Transmission electron microscopy (TEM) studies were performed on a Jeol 2100 HR microscope; TEM samples were prepared by forming a suspension of the particles with a concentration of between 0.1 and $0.01 \mathrm{wt} \%$ solid fraction. Nanocrystalline powder size distributions were measured with a Malvern Mastersizer.

\section{Results and Discussion}

XRD spectra for the Ti-N and Ti-O based materials are shown in Figure $2 a$ and $2 b$, respectively. As seen, two distinct phases were formed in the plasma reaction zone depending on the working gas used. In the first case (see Figure $2 \mathrm{a}$ ), Ti vapour interaction with the nitrogen gas yielded a mixture of

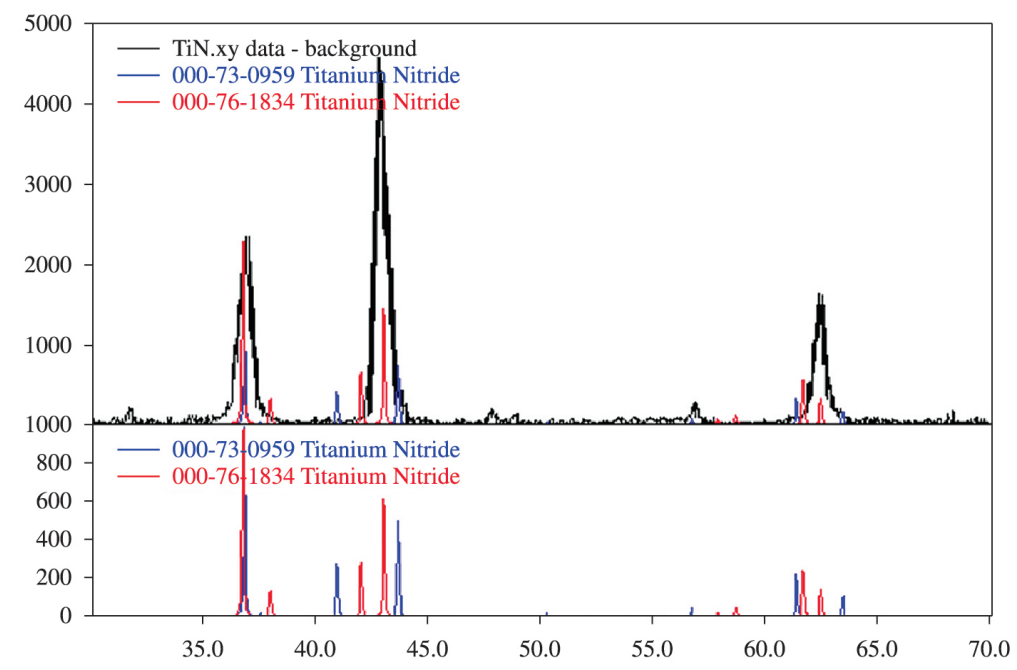

(a)

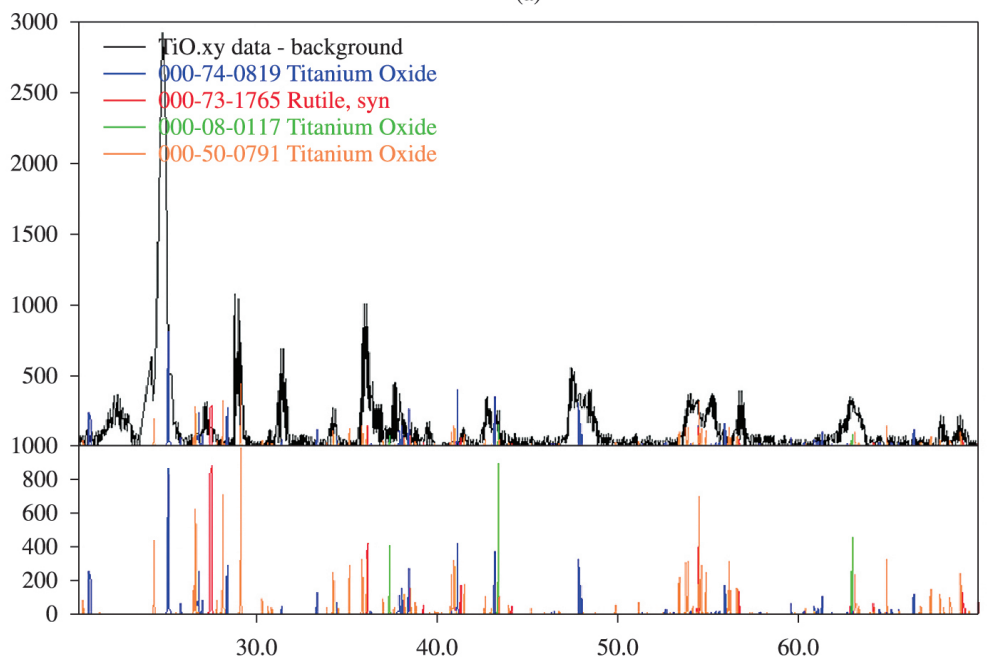

(b)

Figure 2. XRD spectra of the nanosized phases for the a) Ti-N system and b) Ti-O system. 
titanium nitrides. In more detail, the Ti-N peak at 37 degrees was indexed as a mixture of $\mathrm{Ti}_{2} \mathrm{~N}$ and the sub-stoichiometric compound $\mathrm{TiN}_{0.61}$, while 42.89 and 62.51 was mapped to the $\mathrm{TiN}_{0.61}$ phase. On the other hand, the principal Ti-O peak at 24.76 degrees (see Figure 2b) was indexed as a mixture of $\mathrm{Ti}_{3} \mathrm{O}_{5}$ and $\mathrm{Ti}_{9} \mathrm{O}_{17}$, generally off-stoichiometric phases of $\mathrm{TiO}_{2}$ rutile. The rest of the Ti-O peaks were found to be a mixture of stoichiometric rutile and $\mathrm{TiO}$, the latter contributing more towards peaks found higher than 60 degrees 2 theta.

Figure 3 shows EDS of material made both in the presence of 0.5 w.t. $\%$ oxygen as well as in the absence of the oxygen gas. From the EDS spectra, it became evident that

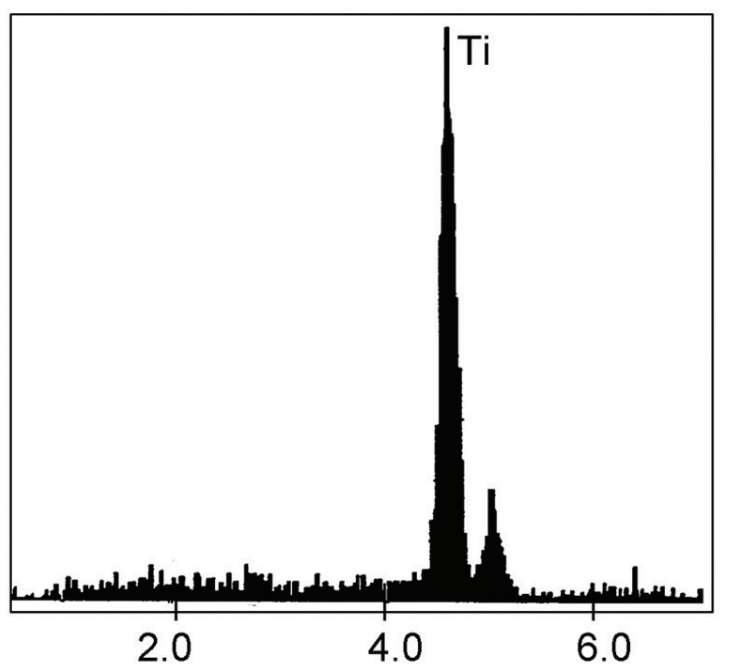

(a)

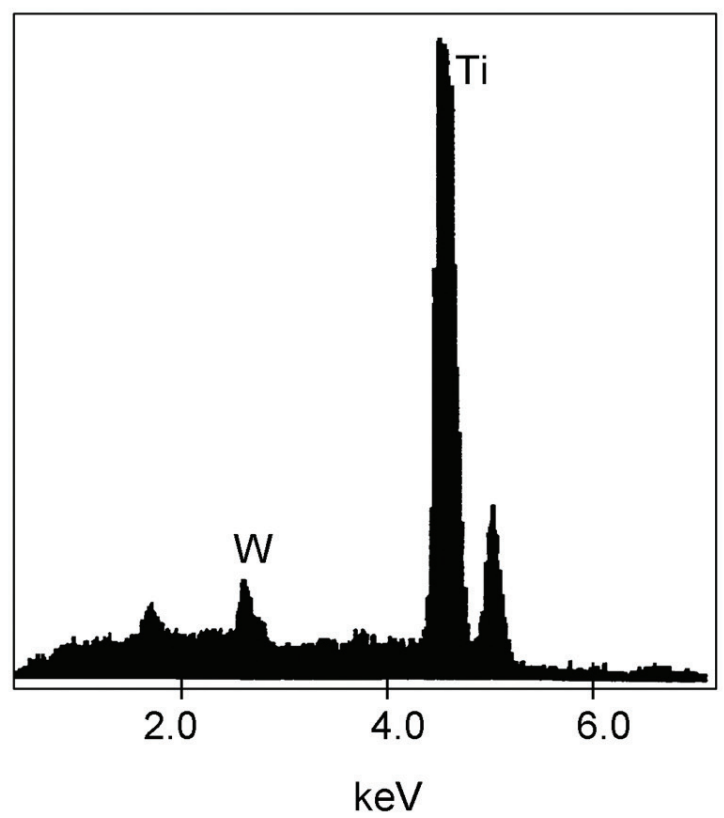

(b)

Figure 3. EDS spectra for the generated nanogranules by a) nitrogen and b) oxygen. even fractional oxygen concentrations may cause tungsten impurities at levels of roughly 1.0 w.t. $\%$ in the intermetallic phases. A typical dispersion of Ti-N intermetallics is shown in Figure 4.

Ti plasma-generated vapour reacting solely with nitrogen formed TiN nanocrystals of average size in the range $8-25 \mathrm{~nm}$. Similarly, the Ti vapour interacting with oxygen gas formed $\mathrm{TiO}_{2}$ nanocrystals (also see XRD analysis) of average sizes in the range 5-40 nm. Figure 5a shows the agglomerate particle size versus the gas flow injected into the nozzle under the applied back pressure, where it can be seen that (due to compressibility of the gas phase) the overall trend is one of decreasing particle size with increasing flowrate. As explained elsewhere ${ }^{19,20}$, there is a transition of the liquid globule break up mechanisms with increasing density (or speed) of the gas phase from the 'bag' to 'the stripping' mode; this transition is in effect revealed by the change of curvature of the plot in Figure 5a. Also, in the Ti vapour/gas two-phase flow, there was a minimum achievable size, of the order of $15 \mathrm{~nm}\left(\mathrm{~d}_{50}\right.$ mass median size), regardless of the increase in the speed of the gas phase, again as shown by the tailing off of the plot in Figure 5a. The distribution of particle sizes for the case of the Ti-N system is presented in Figure 5b, as measured by the size analyser. Overall, the effect of the radial injection point of the gas upon the nanocrystalline particle diameter followed the gas density and was on par with gas compressibility as previously calculated in the case of two-phase flows ${ }^{17-19,21}$. When argon was used as the working gas and oxygen as the reaction gas, an oxygendeficient oxide nanocrystal was formed, as detected by X-ray diffraction (see Figure 1a), while sub-stoichiometric material required further processing for complete oxidation; such post processing involved annealing of the powders in ambient conditions. This step was often found increase the degree of agglomeration and also caused an increase of

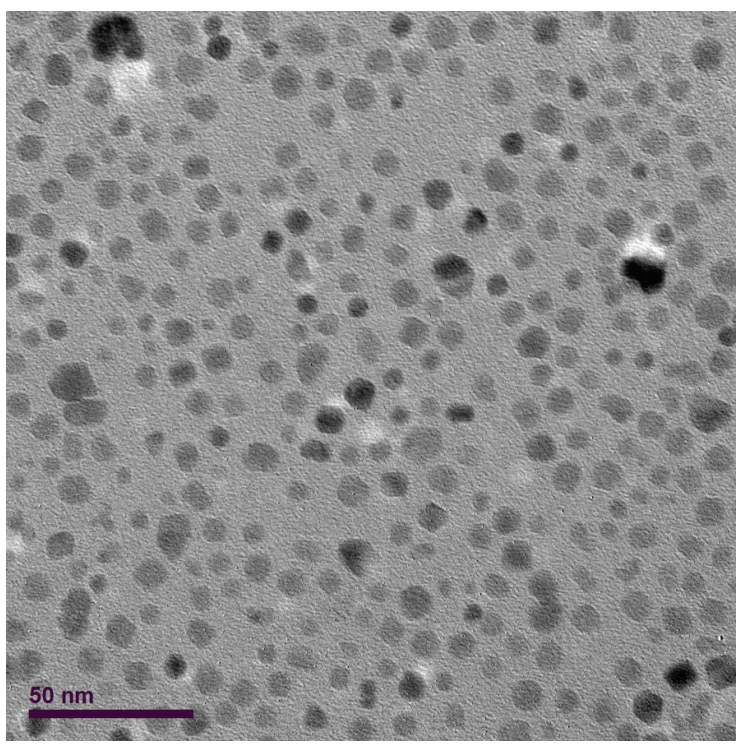

Figure 4. Representative TEM micrograph of the titanium nitride phase (shown in dark-grey/black colour). 


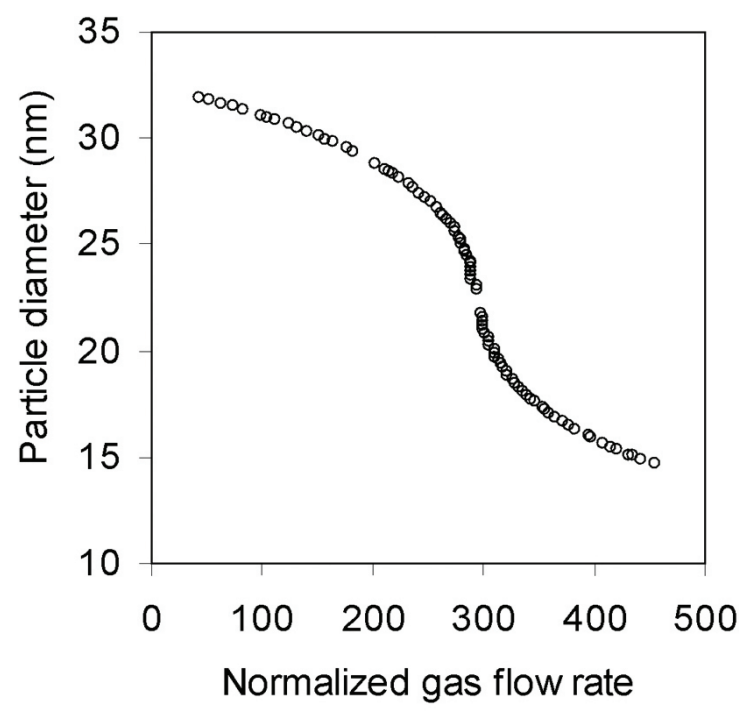

(a)

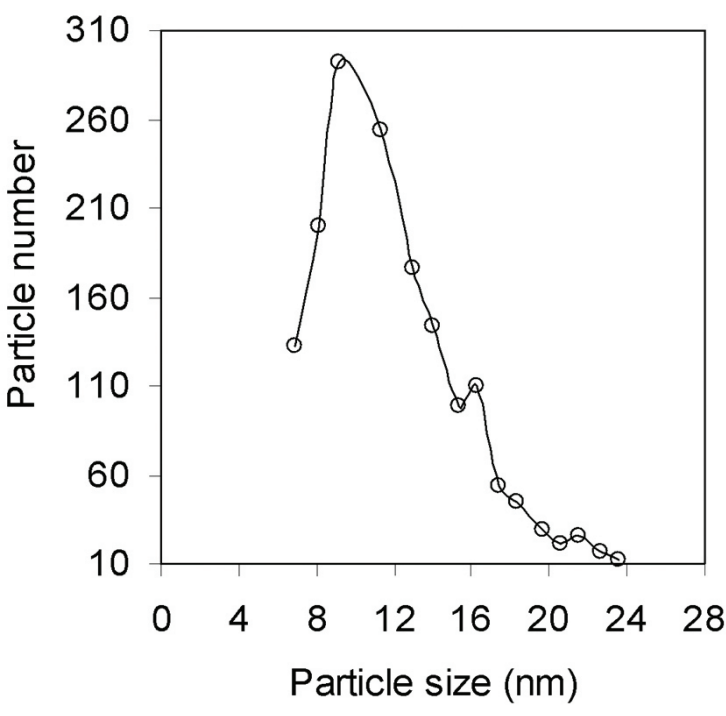

(b)

Figure 5. a) Particle average size vs. gas flowrate and b) mass median size distribution of the sub- $25 \mathrm{~nm}$ particle fraction.

particle size. Agglomeration appeared rather sensitive to the exact gas injection point inside the plasma chamber, as well as to the type of gas injected and to the gas flow rate. On the other hand, the presence of hydrogen in the working gas invariably led to the formation of water vapours and caused oxidation of the resulting nanoparticles. Further cooling led to the formation of metal-oxide ceramic particles due to the presence of oxygen and the high temperatures involved. Air quenching by a flow of $36 \mathrm{~m}^{3} / \mathrm{h}$ added to cool the particles were also observed to prevent agglomeration. The nanocrystalline powders collected were typically

\section{References}

1. Dion I, Rouais F, Trut L, Baquey C, Monties JR and Havlik P. TiN coating: surface characterization and haemocompatibility. Biomaterials. 1993; 14(3):169-176. http://dx.doi.org/10.1016/0142-9612(93)90019-X

2. Gispert MP, Serro AP, Colaço R, Rego AMB, Alves E, Silva $\mathrm{RC}$ et al. Tribological behaviour of Cl-implanted TiN coatings for biomedical applications. Wear. 2007; 262(11-12):13371345. http://dx.doi.org/10.1016/j.wear.2007.01.017

3. Avasarala B and Haldar P. Durability and degradation mechanism of titanium nitride based electrocatalysts for PEM (proton exchange membrane) fuel cell applications. Energy. 2013; 57:545-553. http://dx.doi.org/10.1016/j. energy.2013.05.021

4. Serro AP, Completo C, Colaço R, Santos F, Silva CL, Cabral JMS et al. A comparative study of titanium nitrides, TiN, TiNbN and TiCN, as coatings for biomedical applications. Surface and Coatings Technology. 2009; 203(24):3701-3707. http://dx.doi. org/10.1016/j.surfcoat.2009.06.010

5. Lackner JM, Waldhauser W and Ebner R. Large-area high-rate pulsed laser deposition of smooth TiCxN1-x coatings at room temperature: mechanical and tribological properties. Surface found to have primary aggregate sizes of $50 \mathrm{~nm}$ and typical agglomerate sizes are $100 \mathrm{~nm}$ (also see Figure 4).

\section{Conclusions}

- Plasma spraying of Ti-based vapors yielded nanogranules. Nanogranule stoichiometry was difficult to control and ranged substantially.

- The synthesis method may provide the basis for larger production as compared to sheer laboratory-grade methods.

and Coatings Technology. 2004; 188-189:519-524. http:// dx.doi.org/10.1016/j.surfcoat.2004.07.009

6. Pham V-H, Jun S-H, Kim H-E and Koh Y-H, Deposition of titanium nitride (TiN) on $\mathrm{Co}-\mathrm{Cr}$ and their potential application as vascular stent. Applied Surface Science. 2012; 258(7):28642868. http://dx.doi.org/10.1016/j.apsusc.2011.10.149

7. Guler U, Naik G, Boltasseva A, Shalaev V and Kildishev A. Nitrides as alternative materials for localized surface plasmon applications. In: Frontiers in Optics. Optical Society of America; 2012. Paper FTh4A. 2.

8. Naik GV, Saha B, Liu J, Saber SM, Stach E, Irudayaraj J et al. Titanium nitride based metamaterial for applications in the visible. In: CLEO: QELS_Fundamental Science. Optical Society of America; 2013. Paper QTu3A. 7.

9. Giolando DM. Nano-crystals of titanium dioxide in aluminum oxide: a transparent self-cleaning coating applicable to solar energy. Solar Energy. 2013; 97:195-199. http://dx.doi. org/10.1016/j.solener.2013.08.024

10. Rudnev VS, Wybornov S, Lukiyanchuk IV, Staedler T, Jiang X, Ustinov AY et al. Thermal behavior of Ni- and Cu-containing plasma electrolytic oxide coatings on titanium. Applied Surface 
Science. 2012; 258(22):8667-8672. http://dx.doi.org/10.1016/j. apsusc.2012.05.071

11. Wojcieszak D, Domaradzki J, Kaczmarek D, Prociow E, Morawski AW and Janus M. Photoluminescence and Photocatalytic Properties of Nanocrystalline TiO2: Tb Thin Films. Journal of Nano Research. 2012; 18:187-193. http:// dx.doi.org/10.4028/www.scientific.net/JNanoR.18-19.187

12. Yang $G$, Zhuang $H$ and Biswas $P$. Characterization and sinterability of nanophase titania particles processed in flame reactors. Nanostructured Materials. 1996; 7:675-689. http:// dx.doi.org/10.1016/0965-9773(96)00033-5

13. Antipas GSE. The effect of increasing sn content on hightemperature mechanical deformation of an $\mathrm{Mg}-3 \% \mathrm{Cu}-1 \% \mathrm{Ca}$ Alloy. Metals. 2013; 3:337-342. http://dx.doi.org/10.3390/ met3040337

14. Haro-Poniatowski E, Rodríguez-Talavera R, de la Cruz HM, Cano-Corona O and Arroyo-Murillo R. Crystallization of nanosized titania particles prepared by the sol-gel process. Journal of Materials Research. 1994; 9:2102-2108. http:// dx.doi.org/10.1557/JMR.1994.2102

15. Antipas GSE. A review of gas atomization and spray forming phenomenology. Powder Metallurgy. 2013; 56(4):317-330. http://dx.doi.org/10.1179/1743290113Y.0000000057
16. Jiang HG, Lau ML and Lavernia EJ. Grain growth behavior of nanocrystalline Inconel 718 and Ni powders and coatings. Nanostructured Materials. 1998; 10:169-178. http://dx.doi. org/10.1016/S0965-9773(98)00058-0

17. Antipas GSE. Spray forming of al alloys: experiment and theory. Materials Research. 2012; 15:131-135. http://dx.doi. org/10.1590/S1516-14392012005000007

18. Antipas GSE. Liquid column deformation and particle size distribution in gas atomization. International Journal of Computational Materials Science and Surface Engineering. 2011; 4:247-264. http://dx.doi.org/10.1504/ IJCMSSE.2011.042822

19. Antipas GSE. Modelling of the break up mechanism in gas atomization of liquid metals. Part II: the gas flow model. Computational Materials Science. 2009; 46:955-959. http:// dx.doi.org/10.1016/j.commatsci.2009.04.046

20. Antipas GSE. Modelling of the break up mechanism in gas atomization of liquid metals. Part I: the surface wave formation model. Computational Materials Science. 2006; 35:416-422. http://dx.doi.org/10.1016/j.commatsci.2005.03.009

21. Antipas G. Gas atomization of aluminium melts: comparison of analytical models. Metals. 2012; 2:202-210. http://dx.doi. org/10.3390/met2020202 